\title{
Ulam-Hyers stabilities of a differential equation and a weakly singular Volterra integral equation
}

\author{
Ozgur Ege ${ }^{1}$, Souad Ayadi ${ }^{2}$ and Choonkil Park ${ }^{3 *}$ (D)
}

\section{"Correspondence:}

baak@hanyang.ac.kr

${ }^{3}$ Research Institute for Natural

Sciences, Hanyang University, Seoul

04763, Korea

Full list of author information is

available at the end of the article

\section{Springer}

\begin{abstract}
In this work we study the Ulam-Hyers stability of a differential equation. Its proof is based on the Banach fixed point theorem in some space of continuous functions equipped with the norm $\|\cdot\|_{\infty}$. Moreover, we get some results on the Ulam-Hyers stability of a weakly singular Volterra integral equation using the Banach contraction principle in the space of continuous functions $C([a, b])$.
\end{abstract}

MSC: $47 \mathrm{H} 10 ; 54 \mathrm{H} 25 ; 39 \mathrm{~B} 72$

Keywords: Ulam-Hyers stability; Fixed point; Differential equation; Volterra integral equation

\section{Introduction and mathematical preliminaries}

In 1922, Banach gave his famous result,the Banach contraction principle, in the concept of the fixed point theory $[1,2]$. Later, most of the authors introduced many works in various spaces using this fixed point theorem or its generalization. Up to now, several developments have occurred in this area, especially the study of the stability problem of a functional equation. The origin of this subject returns to a question of Ulam [3] in 1940, which was related to the stability of group homomorphisms. Hyers [4] was the first researcher who gave the affirmative partial answer to Ulam's question. This type of stability is known as the Ulam-Hyers stability. It has attracted attention of many authors who have published various results on the stability of some classes of functional equations via fixed point approach. For more details on Ulam-Hyers stability, we recommend [5-15].

This paper concerns the study of the Ulam-Hyers stability of a differential equation and a weakly singular Volterra integral equation. In the first case, given an initial condition and an equation $u^{\prime}=f(\cdot, u)$, where $u$ is defined from $I \subset \mathbb{R}$ into $\mathbb{R}^{n}$, we show that when $f$ is continuous and $k$-Lipschitz for some $k$ and when $u$ takes its values on a certain ball $B$, we get the Ulam-Hyers stability. In the second case, we show that we can prove Ulam-Hyers stability when the kernel is composed of a singular part which verifies certain properties in a space $L^{q},(q>1)$ and a continuous function on a part of $\mathbb{R}^{3}$ with a given certain condition on the third variable.

(c) The Author(s) 2021. This article is licensed under a Creative Commons Attribution 4.0 International License, which permits use, sharing, adaptation, distribution and reproduction in any medium or format, as long as you give appropriate credit to the original author(s) and the source, provide a link to the Creative Commons licence, and indicate if changes were made. The images or other third party material in this article are included in the article's Creative Commons licence, unless indicated otherwise in a credit line to the material. If material is not included in the article's Creative Commons licence and your intended use is not permitted by statutory regulation or exceeds the permitted use, you will need to obtain permission directly from the copyright holder. To view a copy of this licence, visit http://creativecommons.org/licenses/by/4.0/. 
Our paper is organized as follows. First, we give some tools needed in the proofs of our main results which will be given in other sections. In Sect. 2, we deal with UlamHyers stability of a differential equation. In the last section, we use the Banach contraction principle to present the Ulam-Hyers stability of certain nonlinear integral equation of Volterra type, where the kernel contains a singular part. Comparing with many papers such as [16-19] in the literature, we believe that our fixed point approach brings new and different perspective to show that some differential and integral equations have the Hyers-Ulam stability.

Theorem 1.1 ([1,2]) Let $(X, d)$ be a complete metric space and $A: X \rightarrow X$ be a contraction, i.e., there exists $\lambda \in[0,1)$ such that

$$
d(A u, A v) \leq \lambda d(u, v), \quad \forall u, v \in X .
$$

Then there exists a unique element $u_{0} \in X$ such that $A u_{0}=u_{0}$. Moreover, $\lim _{n \rightarrow \infty} A^{n} u=u_{0}$ and

$$
d\left(u_{0}, u\right) \leq \frac{1}{1-\lambda} d(u, A u), \quad \forall u \in X .
$$

Definition $1.2([20,21])$ Let $(X, d)$ be a metric space and $A: X \rightarrow X$ be an operator. The fixed point equation

$$
A u=u
$$

is said to be generalized Ulam-Hyers stable if there exists an increasing function $\phi: \mathbb{R}^{+} \rightarrow$ $\mathbb{R}^{+}$continuous in 0 with $\phi(0)=0$ such that, for each $\alpha>0$ and for each mapping $u$ satisfying

$$
d(u, A u) \leq \alpha,
$$

there exists a solution $u_{0}$ of (1.1) such that

$$
d\left(u, u_{0}\right) \leq \phi(\alpha) .
$$

If $\phi(x)=a x$ for each $x \in \mathbb{R}^{+}$, then equation (1.1) is called Ulam-Hyers stable.

Definition 1.3 ([22]) A function $f: \mathbb{A} \rightarrow \mathbb{R}^{m}, \mathbb{A} \subset \mathbb{R}^{n}$ is said to be $k$-Lipschitz, $k \geq 0$, if

$$
|f(a)-f(b)| \leq k|a-b|
$$

for every pair of points $a, b \in \mathbb{A}$. We say that a function is Lipschitz if it is $k$-Lipschitz for some $k$.

Definition 1.4 ([2]) A function $f: \mathbb{A} \rightarrow \mathbb{R}^{m}, \mathbb{A} \subset \mathbb{R} \times \mathbb{R}^{m},(x, u) \mapsto f(x, u)$ is said to be locally Lipschitz with respect to $u$ if, for all $\left(x_{0}, u_{0}\right) \in \mathbb{A}$, there exist a neighborhood $V$ of $\left(x_{0}, u_{0}\right)$ in $\mathbb{A}$ and a constant $k \geq 0$ such that, for all $(x, u),\left(x, u^{\prime}\right) \in V$, we have

$$
\left|f(x, u)-f\left(x, u^{\prime}\right)\right| \leq k\left|u-u^{\prime}\right|
$$




\section{Ulam-Hyers stability of a differential equation}

Our result in this section concerns the Ulam-Hyers stability for the differential equation

$$
u^{\prime}=f(x, u), \quad(x, u) \in U, x_{0} \in I, \quad u\left(x_{0}\right)=y_{0},
$$

where $\left(x_{0}, y_{0}\right) \in U, u: I \subset \mathbb{R} \rightarrow \mathbb{R}^{n}$ is a function, $U$ is an open set in $\mathbb{R} \times \mathbb{R}^{n}$, and $f: U \rightarrow \mathbb{R}^{n}$ is a continuous function.

Let $V$ be a neighborhood of $\left(x_{0}, y_{0}\right)$. Assume that $f$ is a $k$-Lipschitz with respect to $u$ on $V$. Let $T_{0}>0, r_{0}>0$, and $C_{0}=\left[x_{0}-T_{0}, x_{0}+T_{0}\right] \times B\left(y_{0}, r_{0}\right) \subset V$, where $B\left(y_{0}, r_{0}\right)$ is the closed ball. One can easily see that $f$ is bounded on the compact set $C_{0}$ and $\rho=\sup _{(x, u) \in C_{0}}|f(x, u(x))|$.

Let $\mu=\min \left(T_{0}, \frac{r_{0}}{\rho}\right)$, where $r_{0}$ and $T_{0}$ are chosen so that $0<\mu<\frac{1}{k}$. Denote by $F$ the set of continuous functions on $\left[x_{0}-\mu, x_{0}+\mu\right] \times B\left(y_{0}, r_{0}\right)$ and equip this set with the usual $\|\cdot\|_{\infty}$ norm.

Theorem 2.1 Let $\varepsilon$ be a positive real number. If $u \in F$ is a function such that

$$
\left\|u^{\prime}(x)-f(x, u(x))\right\|_{\infty} \leq \varepsilon, \quad u\left(x_{0}\right)=y_{0},
$$

then there exist $\theta_{0}>0$ and unique $u_{0} \in F$ such that

$$
u_{0}^{\prime}(x)=f\left(x, u_{0}(x)\right), \quad u_{0}\left(x_{0}\right)=y_{0}
$$

and

$$
\left\|u(x)-u_{0}(x)\right\|_{\infty} \leq \theta_{0} \varepsilon, \quad \forall x \in\left[x_{0}-\mu, x_{0}+\mu\right] .
$$

That is, the differential equation (2.1) is locally Ulam-Hyers stable.

Proof The proof will be given in four steps.

- Step 1:

Consider the operator $T: F \rightarrow F$ defined by

$$
T u(x)=y_{0}+\int_{x_{0}}^{x} f(s, u(s)) d s .
$$

$T$ is well defined. Indeed, let $u \in F$ for any $x \in\left[x_{0}-\mu, x_{0}+\mu\right]$. Then we have

$$
\begin{aligned}
\left\|T u(x)-y_{0}\right\|_{\infty} & =\left\|\int_{x_{0}}^{x} f(s, u(s)) d s\right\|_{\infty} \\
& \leq \int_{x_{0}}^{x}\|f(s, u(s))\|_{\infty} d s \\
& \leq \rho \int_{x_{0}}^{x} d s \\
& \leq \rho\left(x-x_{0}\right) \\
& \leq r_{0} .
\end{aligned}
$$


That is, $T u(x) \in B\left(y_{0}, r_{0}\right)$.

- Step 2:

Let $u \in F$. We have the following equivalence:

$u$ is a solution of equation $(2.1) \Longleftrightarrow u$ is a fixed point of the operator $T$.

We will show this equivalence.

$(\Rightarrow)$ If $u$ is a solution of the differential equation (2.1), since the function $s \mapsto f(s, u(s))$ is continuous, we can integrate with respect to $s$ the equation $u^{\prime}(s)=f(s, u(s)), s \in\left[x_{0}-\mu, x_{0}+\mu\right]$. Then we have

$$
\int_{x_{0}}^{x} f(s, u(s)) d s=\int_{x_{0}}^{x} u^{\prime}(s) d s=u(x)-u\left(x_{0}\right)=u(x)-y_{0},
$$

which means that $T u=u$.

$(\Leftarrow)$ Now, we suppose that $u$ is a fixed point of the operator $T$. Then

$$
T u=u \quad \text { and } \quad y_{0}+\int_{x_{0}}^{x} f(s, u(s)) d s=u(x), \quad \forall x \in\left[x_{0}-\mu, x_{0}+\mu\right] .
$$

Since $f$ is continuous on $U, u$ is also continuous on $U$ and differentiable and $u^{\prime}(x)=f(x, u(x))$. Moreover,

$$
u\left(x_{0}\right)=y_{0}+\int_{x_{0}}^{x_{0}} f(s, u(s)) d s=y_{0}
$$

- Step 3:

In this step, we prove that the operator $T$ is a contraction. Let $u, v \in F$. Then we have

$$
\begin{aligned}
\|T u(x)-T v(x)\|_{\infty} & =\left\|\int_{x_{0}}^{x}(f(s, u(s))-f(s, v(s))) d s\right\|_{\infty} \\
& \leq \int_{x_{0}}^{x}\|f(s, u(s))-f(s, v(s))\|_{\infty} d s \\
& \leq k \int_{x_{0}}^{x}\|u(s)-v(s)\|_{\infty} d s \\
& \leq k\|u-v\|_{\infty}\left|\int_{x_{0}}^{x} d s\right| \\
& \leq k\|u-v\|_{\infty}\left|x-x_{0}\right| .
\end{aligned}
$$

Taking the sup on $x$ over $\left[x_{0}-\mu, x_{0}+\mu\right]$, we get

$$
\|T u-T v\|_{\infty} \leq k \mu\|u-v\|_{\infty},
$$

that is,

$$
\|T u-T v\|_{\infty} \leq \lambda\|u-v\|_{\infty} .
$$


As $0<\lambda=k \mu<1$, then $T$ is a contraction. From Theorem 1.1, there exists a unique element $u^{*} \in F$ such that $T u^{*}=u^{*}$ and

$$
\left\|u^{*}-u\right\|_{\infty}=\frac{1}{1-\lambda}\|T u-u\|_{\infty}, \quad \forall u \in F
$$

Moreover, from Step 2, we find

$$
\left(u^{*}\right)^{\prime}=f\left(x, u^{*}(x)\right) \text { and } u^{*}\left(x_{0}\right)=y_{0} .
$$

- Step 4:

Let $\varepsilon$ be a positive real number and $u$ be a point in $F$ such that

$$
\left\|u^{\prime}(x)-f(x, u(x))\right\|_{\infty} \leq \varepsilon, \quad u\left(x_{0}\right)=y_{0} .
$$

Using (2.7) and integrating with respect to $s$ over $\left[x_{0}-\mu, x_{0}+\mu\right]$, we get

$$
\|u(x)-T u(x)\|_{\infty} \leq \varepsilon \mu, \quad \forall x \in\left[x_{0}-\mu, x_{0}+\mu\right] .
$$

On the other hand, we obtain the following inequalities:

$$
\begin{aligned}
\left\|u^{*}-u\right\|_{\infty} & \leq\left\|u^{*}-T u\right\|_{\infty}+\|T u-u\|_{\infty} \\
& \leq\left\|T u^{*}-T u\right\|_{\infty}+\|T u-u\|_{\infty} \\
& \leq k \mu\left\|u^{*}-u\right\|_{\infty}+\varepsilon \mu,
\end{aligned}
$$

that is,

$$
\begin{aligned}
(1-k \mu)\left\|u^{*}-u\right\|_{\infty} & \leq \varepsilon \mu, \\
\left\|u^{*}-u\right\|_{\infty} & \leq \frac{\mu}{1-k \mu} \varepsilon .
\end{aligned}
$$

Setting $\theta_{0}=\frac{\mu}{1-k \mu}>0$, we have

$$
\left\|u^{*}-u\right\|_{\infty} \leq \theta_{0} \varepsilon
$$

which gives

$$
\left\|u^{*}(x)-u(x)\right\|_{\infty} \leq \theta_{0} \varepsilon, \quad \forall x \in\left[x_{0}-\mu, x_{0}+\mu\right]
$$

That is the needed result.

Example 2.2 Let $\xi \neq 0$ be a positive real number. Consider the following function:

$$
f(x, u(x))=-\frac{1}{\xi} \sin \left(\frac{x}{\xi}\right) \quad \text { with } u(x)=\frac{2}{\xi} \cos \left(\frac{x}{\xi}\right) .
$$

Since

$$
\left|u^{\prime}(x)-f(x, u(x))\right|=\left|-\frac{2}{\xi} \sin \left(\frac{x}{\xi}\right)+\frac{1}{\xi} \sin \left(\frac{x}{\xi}\right)\right| \leq \frac{1}{\xi},
$$


we have $\left|u^{\prime}(x)-f(x, u(x))\right| \rightarrow 0$ when $\xi \rightarrow \infty$. So condition (2.7) is satisfied. From Theorem 2.1, the differential equation $u^{\prime}=f(x, u(x))$ is locally Hyers-Ulam stable.

\section{Ulam-Hyers stability of a nonlinear Volterra integral equation}

In this section, we are interested in the study of Ulam-Hyers stability of a nonlinear Volterra integral equation whose kernel contains a weakly singular part as follows:

$$
\varphi(x)=f(x)+\int_{a}^{x} \vartheta(x-t) K(x, t, \varphi(t)) d t ; \quad \forall x \in[a, b],
$$

where $\varphi \in C([a, b])$ and $K$ is a function defined by

$$
\begin{aligned}
K:[a, b] \times[a, b] \times \mathbb{R} & \longrightarrow \mathbb{R} \\
(x, t, z) & \longmapsto K(x, t, z) .
\end{aligned}
$$

Let us consider the operator $T: C([a, b]) \rightarrow C([a, b])$ defined by

$$
(T \varphi)(x)=f(x)+\int_{a}^{x} \vartheta(x-t) K(x, t, \varphi(t)) d t, \quad x \in[a, b]
$$

and assume that the following hypotheses are satisfied:

(1) $K \in C\left([a, b]^{2} \times \mathbb{R}\right)$,

(2) $\exists \sigma>0, \forall x, t \in[a, b], \forall u \in \mathbb{R},|K(x, t, u)| \leq \sigma$,

(3) $H(x, t)=\vartheta(x-t) \in C\left([a, b]^{2}\right), \forall x \neq t$, where $H:[a, b] \rightarrow[a, b]$ is a function,

(4) $f \in C([a, b])$,

(5) there exists $M>0$ such that $\forall x, t \in[a, b], \forall u, v \in \mathbb{R}$

$$
|K(x, t, u)-K(x, t, v)| \leq M|u-v|,
$$

(6) for $q>1$, there exists $\rho>0$ such that $H(x, t) \in L^{q}([a, b])$ and

$$
\|H(x, t)\|_{q} \leq \rho, \quad \forall x, t \in[a, b], x \neq t .
$$

Proposition 3.1 Under the above assumptions, the operator $T$ is well defined.

Proof Let $x, y \in[a, b]$. Our goal is to show that

$$
\lim _{x \rightarrow y}|(T \varphi)(x)-(T \varphi)(y)|=0 .
$$

For this purpose, we consider the following statements:

$$
\begin{aligned}
|(T \varphi)(x)-(T \varphi)(y)|= & \left|\int_{a}^{x} \vartheta(x-t) K(x, t, \varphi(t)) d t-\int_{a}^{y} \vartheta(y-t) K(y, t, \varphi(t)) d t\right| \\
\leq & \left|\int_{a}^{x} \vartheta(x-t) K(x, t, \varphi(t)) d t-\int_{a}^{y} \vartheta(y-t) K(y, t, \varphi(t)) d t\right| \\
& +|f(x)-f(y)|,
\end{aligned}
$$




$$
\begin{aligned}
& \int_{a}^{x} \vartheta(x-t) K(x, t, \varphi(t)) d t-\int_{a}^{y} \vartheta(y-t) K(y, t, \varphi(t)) d t \\
& =\int_{a}^{x-\epsilon} \vartheta(x-t) K(x, t, \varphi(t)) d t+\int_{x-\epsilon}^{x} \vartheta(x-t) K(x, t, \varphi(t)) d t \\
& \quad-\int_{a}^{x-\epsilon} \vartheta(y-t) K(y, t, \varphi(t)) d t-\int_{x-\epsilon}^{y-\epsilon} \vartheta(y-t) K(y, t, \varphi(t)) d t \\
& \quad-\int_{y-\epsilon}^{y} \vartheta(y-t) K(y, t, \varphi(t)) d t+\int_{a}^{x-\epsilon} \vartheta(y-t) K(x, t, \varphi(t)) d t \\
& \quad-\int_{a}^{x-\epsilon} \vartheta(y-t) K(x, t, \varphi(t)) d t,
\end{aligned}
$$

that is,

$$
\begin{aligned}
\int_{a}^{x} \vartheta & (x-t) K(x, t, \varphi(t)) d t-\int_{a}^{y} \vartheta(y-t) K(y, t, \varphi(t)) d t \\
= & \int_{a}^{x-\epsilon}(\vartheta(x-t)-\vartheta(y-t)) K(x, t, \varphi(t)) d t \\
& \quad+\int_{x-\epsilon}^{x} \vartheta(x-t) K(x, t, \varphi(t)) d t-\int_{a}^{x-\epsilon} \vartheta(y-t)(K(x, t, \varphi(t))-K(y, t, \varphi(t))) d t \\
& \quad-\int_{x-\epsilon}^{y-\epsilon} \vartheta(y-t) K(y, t, \varphi(t)) d t-\int_{y-\epsilon}^{y} \vartheta(y-t) K(y, t, \varphi(t)) d t .
\end{aligned}
$$

Taking the absolute value from both sides of the above equation, we get the following:

$$
\begin{aligned}
& \left|\int_{a}^{x} \vartheta(x-t) K(x, t, \varphi(t)) d t-\int_{a}^{y} \vartheta(y-t) K(y, t, \varphi(t)) d t\right| \\
& \leq\left|\int_{a}^{x-\epsilon}(\vartheta(x-t)-\vartheta(y-t)) K(x, t, \varphi(t)) d t\right| \\
& \quad+\left|\int_{x-\epsilon}^{x} \vartheta(x-t) K(x, t, \varphi(t)) d t\right|+\left|\int_{a}^{x-\epsilon} \vartheta(y-t)(K(x, t, \varphi(t))-K(y, t, \varphi(t))) d t\right| \\
& \quad+\left|\int_{x-\epsilon}^{y-\epsilon} \vartheta(y-t) K(y, t, \varphi(t)) d t\right|+\left|\int_{y-\epsilon}^{y} \vartheta(y-t) K(y, t, \varphi(t)) d t\right| .
\end{aligned}
$$

Moreover, we obtain

$$
\begin{aligned}
\left|\int_{x-\epsilon}^{x} \vartheta(x-t) K(x, t, \varphi(t)) d t\right| & \leq \int_{x-\epsilon}^{x}|\vartheta(x-t) K(x, t, \varphi(t))| d t \\
& \leq \int_{x-\epsilon}^{x}|\vartheta(x-t)||K(x, t, \varphi(t))| d t \\
& \leq \sigma \int_{x-\epsilon}^{x}|\vartheta(x-t)| d t \\
& \leq \sigma \int_{x-\epsilon}^{x}\left(|\vartheta(x-t) d t|^{q}\right)^{\frac{1}{q}}\left(\int_{x-\epsilon}^{x}(1)^{p} d t\right)^{\frac{1}{p}} \\
& \leq \sigma\|\vartheta(x-t)\|_{q}|\epsilon|^{\frac{1}{p}} \\
& \leq \sigma \rho \epsilon^{\frac{1}{p}}
\end{aligned}
$$


when $x \rightarrow y$, that is, $\exists \epsilon>0,|x-y| \leq \epsilon$. In the same manner, we get

$$
\left|\int_{y-\epsilon}^{y} \vartheta(y-t) K(y, t, \varphi(t)) d t\right| \leq \sigma \rho \epsilon_{*}^{\frac{1}{p}}
$$

and

$$
\begin{aligned}
& \left|\int_{x-\epsilon}^{y-\epsilon} \vartheta(y-t) K(y, t, \varphi(t)) d t\right| \leq \sigma \rho \epsilon_{\star}^{\frac{1}{p}}, \\
& \left|\int_{a}^{x} \vartheta(y-t)(K(x, t, \varphi(t))-K(y, t, \varphi(t))) d t\right| \\
& \quad \leq \int_{a}^{x-\epsilon}|\vartheta(y-t)(K(x, t, \varphi(t))-K(y, t, \varphi(t)))| d t \\
& \quad \leq \int_{a}^{x-\epsilon}|\vartheta(y-t) \|(K(x, t, \varphi(t))-K(y, t, \varphi(t)))| d t \\
& \quad \leq\|\vartheta(x-t)\|\left\|_{q}\right\| K(x, t, \varphi(t))-K(y, t, \varphi(t)) \|_{p} \\
& \quad \leq \rho \gamma\|K(x, t, \varphi(t))-K(y, t, \varphi(t))\|_{\infty} .
\end{aligned}
$$

As $K$ is continuous with respect to the first variable, we have

$$
\forall \varepsilon>0, \quad \exists \epsilon_{0}>0:|x-y|<\epsilon_{0} \quad \Longrightarrow \quad|K(x, t, \varphi(t))-K(y, t, \varphi(t))| \leq \varepsilon .
$$

Then we obtain

$$
\left|\int_{a}^{x-\epsilon} \vartheta(y-t)(K(x, t, \varphi(t))-K(y, t, \varphi(t))) d t\right| \leq \rho \varepsilon \quad \text { for }|x-y|<\epsilon_{0} .
$$

On the other hand, we have

$$
\begin{aligned}
\left|\int_{a}^{x-\epsilon}(\vartheta(x-t)-\vartheta(y-t)) K(x, t, \varphi(t)) d t\right| & \leq \int_{a}^{x}|(\vartheta(x-t)-\vartheta(y-t)) K(x, t, \varphi(t))| d t \\
& \leq \int_{a}^{x}|(\vartheta(x-t)-\vartheta(y-t))||K(x, t, \varphi(t))| d t \\
& \leq \sigma \int_{a}^{x}|(\vartheta(x-t)-\vartheta(y-t))| d t .
\end{aligned}
$$

Since $H:\left[x-\frac{\epsilon}{2}, b\right] \times[a, x-\epsilon]$ is continuous, we conclude that

$$
\forall \varepsilon>0, \exists \epsilon_{1}>0,|x-y|<\varepsilon_{1} \quad \Rightarrow \quad|H(x, t)-H(y, t)|<\frac{\varepsilon}{b-a} .
$$

So

$$
\begin{aligned}
\int_{a}^{x-\epsilon}|H(x, t)-H(y, t)| d t & =\int_{a}^{x-\epsilon}|\vartheta(x-t)-\vartheta(y-t)| d t \\
& \leq \frac{\varepsilon}{b-a}|x-\epsilon-a| \\
& \leq \varepsilon
\end{aligned}
$$


From $x-\frac{\epsilon}{2}<b$, we have $x-\epsilon<b-\frac{\epsilon}{2}<b$ and $x-\varepsilon<b-a$. For $|x-y|<\min \left(\epsilon, \epsilon_{0}, \epsilon_{1}, \epsilon_{*}, \epsilon_{\star}\right)$, we have

$$
\lim _{x \rightarrow y}|(T \varphi)(x)-(T \varphi)(y)|=0
$$

which means that $(T \varphi) \in C([a, b])$, that is, $T$ is well defined.

Theorem 3.2 Let $\varepsilon$ be a positive real number. If $\varphi \in C([a, b])$ is given such that

$$
\left|\varphi(x)-f(x)-\int_{a}^{x} \vartheta(x-t) K(x, t, \varphi(t)) d t\right| \leq \varepsilon, \quad x \in[a, b],
$$

then there exist $\theta_{0}>0$ and unique $\varphi_{0} \in C([a, b])$ such that

$$
\varphi_{0}(x)=f(x)+\int_{a}^{x} \vartheta(x-t) K\left(x, t, \varphi_{0}(t)\right) d t
$$

and

$$
\left|\varphi(x)-\varphi_{0}(x)\right| \leq \theta_{0} \varepsilon, \quad x \in[a, b] .
$$

Proof The operator $T: C([a, b]) \rightarrow C([a, b])$ defined by

$$
(T \varphi)(x)=f(x)+\int_{a}^{x} \vartheta(x-t) K(x, t, \varphi(t)) d t, \quad x \in[a, b],
$$

is a contraction in the metric space $\left(X, d_{\tau}\right)$, where $X=C([a, b])$ and $d_{\tau}$ the Bielecki metric

$$
d_{\tau}(\varphi, \psi)=\sup _{x \in[a, b]} \frac{|\varphi(x)-\psi(x)|}{e^{\tau(x-a)}}, \quad \forall \varphi, \psi \in X
$$

where $\tau$ is chosen such that

$$
\left(\tau\left(\frac{q}{q-1}\right)\right)^{\frac{q-1}{q}}>M \rho .
$$

Indeed,

$$
\begin{aligned}
& |T \varphi(x)-T \psi(x)| \\
& \quad=\left|f(x)+\int_{a}^{x} \vartheta(x-t) K(x, t, \varphi(t)) d t-f(x)-\int_{a}^{x} \vartheta(x-t) K(x, t, \psi(t)) d t\right| \\
& \quad \leq \int_{a}^{x}|\vartheta(x-t)(K(x, t, \varphi(t))-K(x, t, \psi(t)))| d t \\
& \quad \leq M \int_{a}^{x}|\vartheta(x-t)||\varphi(t)-\psi(t)| d t \\
& \quad \leq M \sup _{x \in[a, b]} \frac{|\varphi(x)-\psi(x)|}{e^{\tau(x-a)}} \int_{a}^{x}|\vartheta(x-t)| e^{\tau(t-a)} d t \\
& \quad \leq M d_{\tau}(\varphi, \psi)\left(\int_{a}^{x}|\vartheta(x-t)|^{q} d t\right)^{\frac{1}{q}}\left(\int_{a}^{x}\left|e^{\tau(t-a)}\right|^{p} d t\right)^{\frac{1}{p}}
\end{aligned}
$$




$$
\begin{aligned}
& \leq M d_{\tau}(\varphi, \psi) \rho \frac{1}{(\tau p)^{\frac{1}{p}}}\left(e^{\tau p(x-a)}\right)^{\frac{1}{p}}, \quad \text { with } \frac{1}{p}+\frac{1}{q}=1 \\
& \leq \frac{M \rho}{(\tau p)^{\frac{1}{p}}} d_{\tau}(\varphi, \psi) e^{\tau(x-a)}
\end{aligned}
$$

So

$$
d_{\tau}(T \varphi, T \psi) \leq \frac{M \rho}{(\tau p)^{\frac{1}{p}}} d_{\tau}(\varphi, \psi)
$$

Since $\left(\tau\left(\frac{q}{q-1}\right)\right)^{\frac{q-1}{q}}>M \rho$, we have

$$
\frac{M \rho}{(\tau p)^{\frac{1}{p}}}<1
$$

Set $\theta=\frac{M \rho}{(\tau p)^{\frac{1}{p}}}$ with $p=q-1$. By Theorem 1.1 , there exists unique $\varphi_{0} \in X$ satisfying

$$
T \varphi_{0}=\varphi_{0} \quad \text { and } \quad d_{\tau}\left(\varphi_{0}, \varphi\right) \leq \frac{1}{1-\theta} d_{\tau}(\varphi, T \varphi), \quad \forall \varphi \in X
$$

Now, let $\varepsilon>0$ and $\varphi \in X$ such that

$$
\left|\varphi(x)-f(x)-\int_{a}^{x} \vartheta(x-t) K(x, t, \varphi(t)) d t\right| \leq \varepsilon, \quad x \in[a, b] .
$$

Then

$$
d_{\tau}(\varphi, T(\varphi)) \leq \varepsilon e^{-\tau p(x-a)}, \quad x \in[a, b],
$$

and

$$
d_{\tau}\left(\varphi_{0}, \varphi\right) \leq \frac{1}{1-\theta} \varepsilon e^{-\tau p(x-a)}, \quad x \in[a, b] .
$$

That is,

$$
\left|\varphi_{0}(x)-\varphi(x)\right| \leq \frac{1}{1-\theta} \varepsilon, \quad x \in[a, b],
$$

which means that the integral equation

$$
\varphi(x)=f(x)+\int_{a}^{x} \vartheta(x-t) K(x, t, \varphi(t)) d t ; \quad \forall x \in[a, b]
$$

is Ulam-Hyers stable.

Example 3.3 Let $\sigma$ be a positive real number. Consider the following functions $\varphi, K, H$ such that, for all $x, t \in[a, b], \forall u \in \mathbb{R}$ :

$$
\varphi(x)=\sin (x), \quad K(x, t, u)=\sigma u, \quad f \in C([a, b])
$$




$$
H(x, t)=\frac{1}{(x-t)^{s}} \quad \text { with } x \neq t, 0<s<1 .
$$

It is clear that conditions (1), (3), and (4) in Proposition 3.1 are satisfied. It suffices to show that conditions (2), (5), and (6) in Proposition 3.1 are satisfied.

(2) is satisfied because $\forall x, t \in[a, b]$ and $\forall \varphi(x), \phi(x) \in \mathbb{R}$, we have

$$
|K(x, t, \varphi(x))|=|\sigma \varphi(x)|=|\sigma||\varphi(x)| \leq \sigma .
$$

Since

$$
|K(x, t, \varphi(x))-K(x, t, \phi(x))|=\sigma \mid \varphi(x))-\phi(x)|\leq M| \varphi(x))-\phi(x) \mid
$$

(5) is also satisfied. Let $q>1$. The following inequalities

$$
\left(\int_{a}^{x}\left|\frac{1}{(x-t)^{s}}\right|^{q} d t\right)^{\frac{1}{q}} \leq\left(\frac{(b-a)^{1-s q}}{1-s q}\right)^{\frac{1}{q}} \leq \rho
$$

show that condition (6) is satisfied. By Theorem 3.2, the nonlinear Volterra integral equation

$$
\sin (x)=f(x)+\int_{a}^{x} \frac{1}{(x-t)^{s}} \sigma \sin (t) d t
$$

has the Hyers-Ulam stability.

\section{Conclusion}

In this paper, we have studied the Ulam-Hyers stability of a differential equation. Its proof is based on the Banach fixed point theorem in some space of continuous functions. Moreover, we have obtained some results on the Ulam-Hyers stability of a weakly singular Volterra integral equation using the Banach contraction principle in the space of continuous functions $C([a, b])$.

\section{Acknowledgements}

We would like to express our gratitude to the anonymous referees for their helpful suggestions and corrections.

Funding

Not applicable.

Availability of data and materials

Not applicable.

Competing interests

The authors declare that they have no competing interests.

\section{Authors' contributions}

The authors equally conceived of the study, participated in its design and coordination, drafted the manuscript, participated in the sequence alignment, and read and approved the final manuscript.

\section{Author details}

'Department of Mathematics, Faculty of Science, Ege University, Bornova, 35100, Izmir, Turkey. ${ }^{2}$ Science Department of Matter, Faculty of Science, Djilali Bounaama University, Khemis Miliana, Algeria. ${ }^{3}$ Research Institute for Natural Sciences, Hanyang University, Seoul 04763, Korea. 


\section{Publisher's Note}

Springer Nature remains neutral with regard to jurisdictional claims in published maps and institutional affiliations.

Received: 18 July 2020 Accepted: 7 December 2020 Published online: 22 January 2021

\section{References}

1. Agarwal, R.P., Meehan, M., O'Regan, D.: Fixed Point Theory and Applications. Cambridge University Press, Cambridge (2004)

2. Agarwal, R.P., O'Regan, D., Sahu, D.R.: Fixed Point Theory for Lipschitzian-Type Mappings with Application. Springer, New York (2009)

3. Ulam, S.M.: Problems in Modern Mathematics. Wiley, New York (1964)

4. Hyers, D.H.: On the stability of the linear functional equation. Proc. Natl. Acad. Sci. USA 27, 222-224 (1941)

5. Gachpazan, M., Baghani, O.: Hyers-Ulam stability of Volterra integral equation. Int. J. Nonlinear Anal. Appl. 1, 19-25 (2010)

6. Huang, J., Jung, S., Li, Y:: On the Hyers-Ulam stability of non-linear differential equations. Bull. Korean Math. Soc. 52, 685-697 (2015)

7. Janfada, M., Sadeghi, G.: Stability of the Volterra integrodifferential equation. Folia Math. 18, 11-20 (2013)

8. Jung, S.: Hyers-Ulam stability of linear differential equations of first order. Appl. Math. Lett. 17, 1135-1140 (2004)

9. Miura, T., Miyajima, S., Takahasi, S.E.: A characterization of Hyers-Ulam stability of first order linear differential operators. J. Math. Anal. Appl. 286, 136-146 (2003)

10. Niazi, A.U.K., Wei, J., Rehman, M.U., Jun, D.: Ulam-Hyers stability for nonlinear fractional neutral differential equations Hacet. J. Math. Stat. 48, 157-169 (2019)

11. Sevgin, S., Sevli, H.: Stability of a nonlinear Volterra integro-differential equation via a fixed point approach. J. Nonlinear Sci. Appl. 9, 200-207 (2016)

12. Shah, A.Z.R.: A fixed point approach to the stability of a nonlinear Volterra integrodifferential equation with delay. Hacet. J. Math. Stat. 47, 615-623 (2018)

13. Cadariu, L., Gãvruţa, P., Gãvruţa, L.: Weighted space method for the stability of some nonlinear equations. Appl. Anal. Discrete Math. 6(1), 126-139 (2012)

14. Gãvruţa, P., Gãvruţa, L.: A new method for the generalized Hyers-Ulam-Rassias stability. Int. J. Nonlinear Anal. Appl. $1(2), 11-18(2010)$

15. Jung, S.M.: A fixed point approach to the stability of differential equation $y^{\prime}=F(x, y)$. Bull. Malays. Math. Sci. Soc. (2) $33(1), 47-56(2010)$

16. Sousa, J.V.C., Oliveira, E.C.: Ulam-Hyers stability of a nonlinear fractional Volterra integro-differential equation. Appl. Math. Lett. 81, 50-56 (2018)

17. Zada, A., Ali, W., Farina, S.: Hyers-Ulam stability of nonlinear differential equations with fractional integrable impulses. Math. Methods Appl. Sci. 40, 5502-5514 (2017)

18. Zada, A., Shah, S.O.: Hyers-Ulam stability of first-order nonlinear delay differential equations with fractional integrable impulses. Hacet. J. Math. Stat. 47, 1196-1205 (2018)

19. Mahmudov, I., Al-Khateeb, A.: Existence and Ulam-Hyers stability of coupled sequential fractional differential equations with integral boundary conditions. J. Inequal. Appl. 2019, Article ID 165 (2019)

20. Andras, S., Baricz, A., Pogany, T.: Ulam-Hyers stability of singular integral equations via weakly Picard operators. Fixed Point Theory 17, 21-36 (2016)

21. Rus, I.A.: Remarks on Ulam stability of the operational equations. Fixed Point Theory 10, 305-320 (2009)

22. Lipschitz, R.: De explicatione per series trigonometricas insttuenda functionum unius variablis arbitrariarum, et praecipue earum, quae per variablis spatium finitum valorum maximorum et minimorum numerum habent infintum disquisitio. J. Reine Angew. Math. 63(63), 296-308 (1984)

\section{Submit your manuscript to a SpringerOpen ${ }^{\circ}$ journal and benefit from:}

- Convenient online submission

- Rigorous peer review

- Open access: articles freely available online

- High visibility within the field

- Retaining the copyright to your article

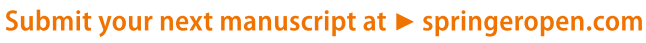

